\title{
Flight Dynamics Analyses of a Propeller-Driven Airplane (I): Aerodynamic and Inertial Modeling of the Propeller
}

\author{
Chang-Joo Kim*, Sang Ho Kim**, TaeSan Park***, Soo Hyung Park**** and Jae Woo Lee***** \\ Department of Aerospace and Information Engineering, Konkuk University, Seoul 143-701, Korea
}

\section{Joon Soo Ko $\mathrm{Ko}^{* * * * *}$}

School of Aerospace and Mechanical Engineering, Korea Aeronautical University, Seoul 143-701, Korea

\begin{abstract}
This paper focuses on aerodynamic and inertial modeling of the propeller for its applications in flight dynamics analyses of a propeller-driven airplane. Unsteady aerodynamic and inertial loads generated by the propeller are formulated using the blade element method, where the local velocity and acceleration vectors for each blade element are obtained from exact kinematic relations for general maneuvering conditions. Vortex theory is applied to obtain the flow velocities induced by the propeller wake, which are used in the computation of the aerodynamic forces and moments generated by the propeller and other aerodynamic surfaces. The vortex lattice method is adopted to obtain the induced velocity over the wing and empennage components and the related influence coefficients are computed, taking into account the propeller induced velocities by tracing the wake trajectory trailing from each of the propeller blades. Aerodynamic forces and moments of the fuselage and other aerodynamic surfaces are computed by using the wind tunnel database and applying strip theory to incorporate viscous flow effects. The propeller models proposed in this paper are applied to predict isolated propeller performances under steady flight conditions. Trimmed level forward and turn flights are analyzed to investigate the effects of the propeller on the flight characteristics of a propeller-driven light-sports airplane. Flight test results for a series of maneuvering flights using a scaled model are employed to run the flight dynamic analysis program for the proposed propeller models. The simulations are compared with the flight test results to validate the usefulness of the approach. The resultant good correlations between the two data sets shows the propeller models proposed in this paper can predict flight characteristics with good accuracy.
\end{abstract}

Key words: propeller-driven airplane, flight dynamics modeling, vortex theory, blade element method

\section{Introduction}

Flight dynamics modeling for a propeller-driven airplane is not an easy task due to the complex flow environments caused by the rotating propeller. In addition, the propeller generates influential gyroscopic moments during maneuvering flights. Furthermore, the wakes trailed from each of the propeller blades generate considerable flow disturbances over the propeller as well as other aerodynamic surfaces [1]. In this paper, propeller modeling techniques are investigated in order to give due consideration to these propeller effects for building an accurate math model. For this purpose, the Level 2 rotor modeling technique, widely used for rotorcraft analyses $[2,3]$, and vortex theory $[4,5]$ are adopted to predict the aerodynamic forces and moments generated by the propeller and to consider the propeller wake effect on other aerodynamic surfaces. Also, the propeller wake theory is combined with the vortex lattice method (VLM) to obtain the induced flow field over the major lifting components including the main wing, the tail wing, fuselage, and control surfaces. The resultant induced velocity at the aerodynamic center of each striped element of the wing is used to compute
This is an Open Access article distributed under the terms of the Creative Commons Attribution Non-Commercial License (http://creativecommons.org/licenses/by$\mathrm{nc} / 3.0 /$ which permits unrestricted non-commercial use, distribution, and reproduction in any medium, provided the original work is properly cited.

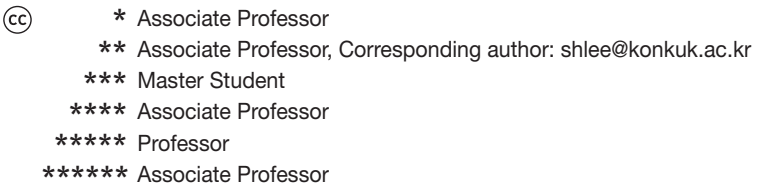


sectional air loads. These air loads are numerically integrated based on the strip theory by using 2-dimensional airfoil aerodynamic tables to ascertain the total air loads of each wing. The viscous drag can then be duly considered in each of the component models, including the propeller. Also, the compressibility effects over the propeller blades can be reflected by applying the blade element method (BEM).

In applying the present method, the mathematical model for flight dynamic analyses is generated for the propellerdriven light airplane using a fixed setting for the root pitch. Aerodynamic tables obtained through wind tunnel tests are used to predict the fuselage aerodynamic forces and moments. Therefore, the resultant math model applies well to the stall angle of attack. The first areas of application of the math model focus on predicting isolated propeller performances in steady flight conditions. Secondly, the propeller effects on flight characteristics are investigated through trim analyses under forward flight conditions. Finally, maneuver simulations are carried out with control inputs used in a series of flight tests and the results obtained using the present math model are compared with those from the flight tests to validate the usefulness of the proposed propeller modeling techniques.

\section{Propeller Model}

The propeller-induced velocity field affects the distribution of aerodynamic forces and moments over the propeller blades. In addition, the trailing propeller wakes disturb the flow field over the fuselage and wing components. Therefore, accurate modeling of the propeller-induced-velocity field over the airplane is extremely important for developing a high-fidelity math model for propeller-driven airplanes. The flow fields generated by the propeller can be predicted at a reasonable computational cost using the lifting-line or lifting-surface theory and vortex wake techniques, which allow both the trailed wake geometry and the resultant disturbed velocity field to be determined. The fuselage and other aerodynamic surfaces are openly immersed in the wake region of the propeller. As a result, these surfaces can experience large variation in relative air velocity depending on the loading conditions of the propeller. In addition, each aerodynamic surface with a lifting force generates an induced velocity field. Therefore, the induced velocities generated by both the propeller and the lifting surfaces should be adequately considered when building an aerodynamic model. For this purpose, the vortex-latticemethod (VLM) is adopted to predict the induced velocities over other aerodynamic surfaces.
In contrast, the approaches mentioned above cannot take into account the viscous and compressible flow effects on the propeller air loads because of their bases in potential flow theory. The compressibility effect on the propeller air loads becomes dominant as the flight speed increases. Also, the viscous drag should be duly considered for an accurate prediction of the propeller performance. These real flow effects can be effectively handled using the BEM, where the aerodynamic coefficients for the blade airfoils, tabulated in the C-81 format, are generally utilized to account for the effects of the Mach number and the angle of attack. Air loads are also numerically integrated to account for these nonlinear aerodynamic effects. The viscous flow effects on the aerodynamic forces and moments of the wing components are considered by applying strip theory and the induced velocities are computed on the aerodynamic centers of the striped wing elements. In addition to the complex aerodynamic effects mentioned above, the propeller can generate discernible gyroscopic moments around the airplane's center of gravity (CG) during an aggressive maneuver. Therefore, the inertial loads of the propeller should also be adequately modeled for flight dynamics analyses and can be accurately predicted using the same BEM framework.

\subsection{Kinematics at the blade element}

For the application of the BEM in computing the inertial and aerodynamic loads of the propeller, the velocity and acceleration at each blade element is derived in an exact manner using the approaches presented in Refs. [6, 7]. For completeness, the main results of the propeller applications are summarized in this section. The inertial acceleration $\ddot{\mathbf{r}}_{C}^{C I}$ and angular motions $\left(\boldsymbol{\omega}_{C}^{C I}, \dot{\boldsymbol{\omega}}_{C}^{C I}\right)$ at the CG of the airplane are traditionally defined using the linear and angular velocity vectors in the body-fixed CG-frame, $\dot{\mathbf{r}}_{C}^{C I}=(u, v, w)^{T}$ and $\boldsymbol{\omega}_{C}^{C I}=(p, q, r)^{T}$, as shown in Eq. (1).

$$
\ddot{\mathbf{r}}_{C}^{C I}=\left(\begin{array}{c}
\dot{u} \\
\dot{v} \\
\dot{w}
\end{array}\right)+\widetilde{\boldsymbol{\omega}}_{C}^{C I} \dot{\mathbf{r}}_{C}^{C I}
$$

The subscript represents the frame (C for CG-fixed frame) used to define vector components, whereas the superscript $C I$ is used to define the vectors at the airplane's CG relative to the center of the inertial reference frame. The matrix expression for the angular velocity $\boldsymbol{\omega}_{C}^{C I}$ is represented by $\widetilde{\boldsymbol{\omega}}_{C}^{C I}$ and similar notational conventions are used in the followon derivations. Using the definition of the airplane's CG position $\mathbf{r}_{C}^{C}$ and the center of the propeller spinner $\mathbf{r}_{C}^{S}$, the linear velocity and acceleration at the spinner center can be 
represented by Eqs (2) and (3) using $\mathbf{r}_{C}^{S C}=\mathbf{r}_{C}^{S}-\mathbf{r}_{C}^{C}$.

$$
\begin{aligned}
& \dot{\mathbf{r}}_{C}^{S I}=\dot{\mathbf{r}}_{C}^{C I}+\widetilde{\boldsymbol{\omega}}_{C}^{C I} \mathbf{r}_{C}^{S C} \\
& \ddot{\mathbf{r}}_{C}^{S I}=\ddot{\mathbf{r}}_{C}^{C I}+\left(\dot{\tilde{\boldsymbol{\omega}}}_{C}^{C I}+\widetilde{\boldsymbol{\omega}}_{C}^{C I} \widetilde{\boldsymbol{\omega}}_{C}^{C I}\right) \mathbf{r}_{C}^{S C}
\end{aligned}
$$

Figure 1 shows the front view of the spinner-fixed nonrotating coordinates $(X, Y, Z)$ with rotational speed $\Omega$ in the counter-clockwise (CCW) direction. In the event that the propeller is installed with the elevation angle $\alpha_{s}$ and the sideward tilt angle $\beta_{s}$ with respect to the CG-fixed frame, the linear velocity and acceleration vectors at the blade element located at the azimuth angle $\psi=\sigma \Omega t(\sigma= \pm 1)$ and the blade radial position $r_{b}$ from the spinner center can be expressed in the rotating spinner reference frame $(x, y, z)$ represented by the subscript $R$ as

$$
\begin{aligned}
\dot{\mathbf{r}}_{R}^{B I}= & \mathbf{L}_{R C} \dot{\mathbf{r}}_{C}^{S I}+r_{b} \widetilde{\boldsymbol{\omega}}_{R}^{R I} \mathbf{e}_{x} \\
\ddot{\mathbf{r}}_{R}^{B I}= & \mathbf{L}_{R C} \ddot{\mathbf{r}}_{C}^{S I}+r_{b}\left(\dot{\widetilde{\boldsymbol{\omega}}}_{R}^{R I}+\widetilde{\boldsymbol{\omega}}_{R}^{R I} \widetilde{\boldsymbol{\omega}}_{R}^{R I}\right) \mathbf{e}_{x} \\
\widetilde{\boldsymbol{\omega}}_{R}^{R I}= & \sigma \Omega \mathbf{L}_{R S} \mathbf{E}_{3} \mathbf{L}_{R S}^{T}+\mathbf{L}_{R C} \widetilde{\boldsymbol{\omega}}_{C}^{C I} \mathbf{L}_{R C}^{T} \\
\dot{\widetilde{\boldsymbol{\omega}}}_{R}^{R I}= & \sigma \dot{\Omega} \mathbf{L}_{R S} \mathbf{E}_{3} \mathbf{L}_{R S}^{T}+\mathbf{L}_{R C} \dot{\widetilde{\boldsymbol{\omega}}}_{C}^{C I} \mathbf{L}_{R C}^{T} \\
& -\sigma \Omega \mathbf{L}_{R S} \mathbf{E}_{3} \mathbf{L}_{S C} \widetilde{\boldsymbol{\omega}}_{C}^{C I} \mathbf{L}_{R C}^{T}+\sigma \Omega \mathbf{L}_{R C} \widetilde{\boldsymbol{\omega}}_{C}^{C I} \mathbf{L}_{S C}^{T} \mathbf{E}_{3} \mathbf{L}_{R S}^{T}
\end{aligned}
$$

Where $\mathbf{L}_{1}, \mathbf{L}_{2}$ and $\mathbf{L}_{3}$ are coordinate transformation matrices related to the angular rotation around the $\mathrm{x}_{-}, \mathrm{y}^{-}$, and $\mathrm{z}$-axes, respectively. Using their angular rotations $\left(\psi, \beta_{s}, \alpha_{s}\right)$, other matrices and the vector $\mathbf{e}_{x}$ are defined as

$$
\begin{aligned}
\mathbf{L}_{R S} & =\mathbf{L}_{3}(\psi) \\
\mathbf{L}_{S P} & =\mathbf{L}_{1}\left(\beta_{S}\right) \mathbf{L}_{2}\left(\alpha_{S}\right) \\
\mathbf{L}_{R C} & =\mathbf{L}_{R S} \mathbf{L}_{S P} \mathbf{L}_{P C}=\mathbf{L}_{3}(\psi) \mathbf{L}_{1}\left(\beta_{S}\right) \mathbf{L}_{2}\left(\alpha_{S}\right) \mathbf{L}_{P C} \\
\mathbf{E}_{j}= & \left(\begin{array}{ccc}
0 & -\delta_{3, j} & \delta_{2, j} \\
\delta_{3, j} & 0 & -\delta_{1, j} \\
-\delta_{2, j} & \delta_{1, j} & 0
\end{array}\right)
\end{aligned}
$$

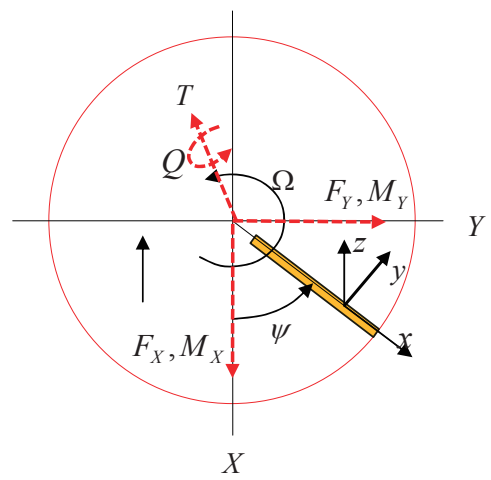

Fig. 1. Notations for the blade element position, propeller forces and moment vectors

$$
\mathbf{e}_{x}=(1,0,0)^{T}
$$

The direction of the propeller rotation is differentiated by $\sigma(\sigma=1$ for CCW, $\sigma=-1$ for clockwise rotation $)$ and $\delta$ represents the Dirac delta function.

\subsection{Propeller forces and moments}

The forces and moments generated by the propeller can be obtained using the BEM by integrating the contributions from all of the blade elements. The aerodynamic forces and moments acting on a blade element can be derived using the velocity vector shown in Eq. (4) with the notations shown in Fig. 2. The geometric pitch angle, the induced angle of attack, and the effective angle of attack are represented by $\theta, \phi$ and $\alpha$, respectively. The relative air velocity at the blade element consists of the inertial velocity defined in Eq. (4) and other contributions such as the induced velocity related to the aerodynamic thrust. If the velocity vector $\dot{\mathbf{r}}_{R}^{B I}$ is split into the tangential, perpendicular, and radial components $\left(U_{T}, U_{P}, U_{R}\right)$ in the rotating spinner reference frame, the aerodynamic forces and moments at the blade element can be computed with aerodynamic tables for the lift, drag, and pitching moment coefficients using the angle of attack and Mach number defined by Eq. (6).

$$
\begin{aligned}
& \begin{array}{ll}
\alpha=\theta-\phi & \text { where }
\end{array} \quad \phi=\tan ^{-1}\left(\frac{U_{P}}{U_{T}}\right) \\
& M=\frac{V}{a} \\
& V=\sqrt{U_{T}^{2}+U_{P}^{2}}
\end{aligned}
$$

Therefore, the six components of averaged aerodynamic forces $\quad \mathbf{F}_{S}^{(\text {aero })}=\left(F_{X}^{(\text {aero })}, F_{Y}^{(\text {aero })}, F_{Z}^{(\text {aero })}\right)^{T}$ and moments $\mathbf{M}_{S}^{(\text {aero })}=\left(M_{X}^{(\text {aero })}, M_{Y}^{(\text {aero })}, M_{Z}^{(\text {aero })}\right)^{T}$ at the spinner center can be obtained for the propeller. Eqs. (7) and (8) show the example formulas for computing $F_{Y}^{(\text {aero })}$ and $M_{Y}^{(\text {aero })}$ when the blade radius is $\mathrm{R}$ and the number of blades is $N_{b}$.

$$
\begin{gathered}
\left.F_{Y}^{(\text {aero })}=-\frac{N_{b}}{4 \pi} \int_{0}^{2 \pi} \int_{0}^{R} \rho V^{2} c\left\{C_{L}(\alpha, M) \sin \phi+C_{D}(\alpha, M) \cos \phi\right\}\right) \sin \psi d r_{b} d \psi \\
M_{Y}^{(\text {aero })}=\frac{N_{b}}{4 \pi} \int_{0}^{2 \pi} \int_{0}^{R} \rho V^{2} c^{2}\left\{C_{M}(\alpha, M) \sin \psi-r_{b}\left(\begin{array}{l}
C_{L}(\alpha, M) \cos \phi \\
-C_{D}(\alpha, M) \sin \phi
\end{array}\right) \cos \psi\right\} d r_{b} d \psi
\end{gathered}
$$

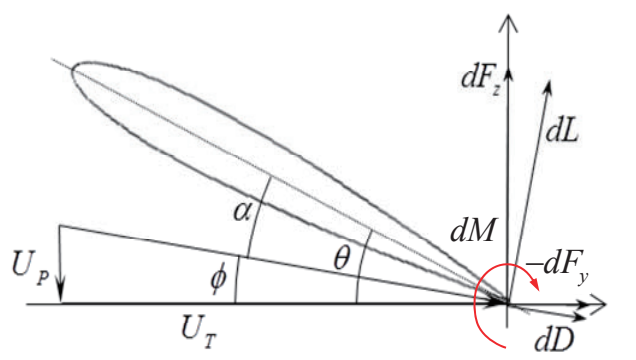

Fig. 2. Aerodynamic parameters at the blade element 
The forces $\mathbf{F}_{S}^{(\text {iner })}$ and moments $\mathbf{M}_{S}^{(\text {iner })}$ due to inertial acceleration can be computed using

$$
\begin{aligned}
& \mathbf{F}_{S}^{(\text {iner })}=-\frac{N_{b}}{2 \pi} \int_{0}^{2 \pi} \int_{0}^{R} m_{b} \mathbf{L}_{R S}^{T} \ddot{\mathbf{r}}_{R}^{B I} d r_{b} d \psi \\
& \mathbf{M}_{S}^{(\text {iner })}=-\frac{N_{b}}{2 \pi} \int_{0}^{2 \pi} \int_{0}^{R} m_{b} r_{b} \mathbf{L}_{R S}^{T}\left(\mathbf{e}_{x} \times \ddot{\mathbf{r}}_{R}^{B I}\right) d r_{b} d \psi
\end{aligned}
$$

where $m_{b}$ represents the blade mass per unit length. The inertial forces and moments in Eq. (9) can be analytically integrated and expressed using the mass properties of the blade, including the total blade mass $M_{b}$ and its polar moments of inertia $J_{b}$. Eqs. (10) and (11) are the resultant equations for the perfectly balanced propeller.

$$
\begin{aligned}
\mathbf{F}_{S}^{(\text {iner })} & =-M_{b} \mathbf{a}_{S} \\
\mathbf{M}_{S}^{(\text {iner })} & =-\frac{J_{b}}{2}\left(\begin{array}{c}
\dot{p}_{S}+\left(r_{S}+\sigma \Omega\right) q_{S} \\
\dot{q}_{S}-\left(r_{S}+\sigma \Omega\right) p_{S} \\
0
\end{array}\right)
\end{aligned}
$$

where $\mathbf{a}_{S}=\mathbf{L}_{S C} \ddot{\mathbf{r}}_{C}^{S I}$ and $\left(p_{S}, q_{S}, r_{S}\right)^{T}=\mathbf{L}_{S C} \widetilde{\boldsymbol{\omega}}_{C}^{C I} \mathbf{L}_{S C}^{T}$

The total forces and moments generated by the propeller can be obtained by summing up the aerodynamic and inertial loads in the non-rotating spinner reference frame as below:

$$
\begin{aligned}
\mathbf{F}_{S} & =\mathbf{F}_{S}^{(\text {aero })}+\mathbf{F}_{S}^{(\text {iner })} \\
\mathbf{M}_{S} & =\mathbf{M}_{S}^{(\text {aero })}+\mathbf{M}_{S}^{(\text {iner })}
\end{aligned}
$$

In the event that the mass properties of the propeller are included in those for the airplane, the gyroscopic moments due to the propeller's rotational speed $\Omega$ should be additionally considered as shown in Eq. (11). It can be demonstrated that those moments cause off-axis responses in the plane normal to the spinning axis. By considering the moment arm to the CG point and the coordinate transformation defined by $\mathbf{L}_{C S}=\mathbf{L}_{P C}^{T} \mathbf{L}_{S P}^{T}$, the forces and moments generated by the propeller can be obtained in the body-fixed CG reference frame.

The aerodynamic coefficients required to compute sectional air loads are tabulated in the standard C-81 format which is widely used in rotorcraft analyses [3]. These coefficients are computed using the Navier-Stokes Solver, KFLOW, which was developed by one of the authors and is widely used in rotorcraft airfoil design [8-10]. The accuracy of KFLOW in predicting the aerodynamic coefficients of the airfoils for rotorcrafts is validated through rigorous correlations of its results with wind tunnel test data. The geometric data for the airfoil sections are measured using the propeller for a light-sports airplane and applied to the KFLOW aerodynamic analyses for airfoils distributed along the blade span. The lift, drag, and pitching moment coefficients of the selected 7 -airfoil sections are tabulated in the C-81 form with variations in Mach numbers $(M=0.1 \sim 0.7)$ and the angles of attack ( $\alpha=-180 \sim 180$ degrees $)$. The results are shown in Fig. 3.

\subsection{Propeller wake model}

The lifting-line theory can be efficiently coupled with the BEM to predict the strength of the line vortex of each blade element using the sectional lift coefficient and relative air speed as shown in Eq. (13).

$$
\Gamma_{b, j}\left(r_{b, j}, \psi\right)=C_{L, j} V_{j} / 2, \quad j=1, \cdots, N_{e}
$$

\section{( $N_{e}$ : the number of blade elements)}

Using these strengths for the bound vortex elements, the structure of the wake trailing from the blades can be modeled using the vortex filaments as shown in Fig. 4. It is assumed that in a short time, a tip vortex is built through the rollup process among the near wake filaments around the blade tip region and originates from the aerodynamic center of the tip airfoil. The wake age (length) $\zeta_{F W}$ of the tip vortex filament is established for a period long enough to cover its influence on the tail parts of the airplane, while relatively short wake
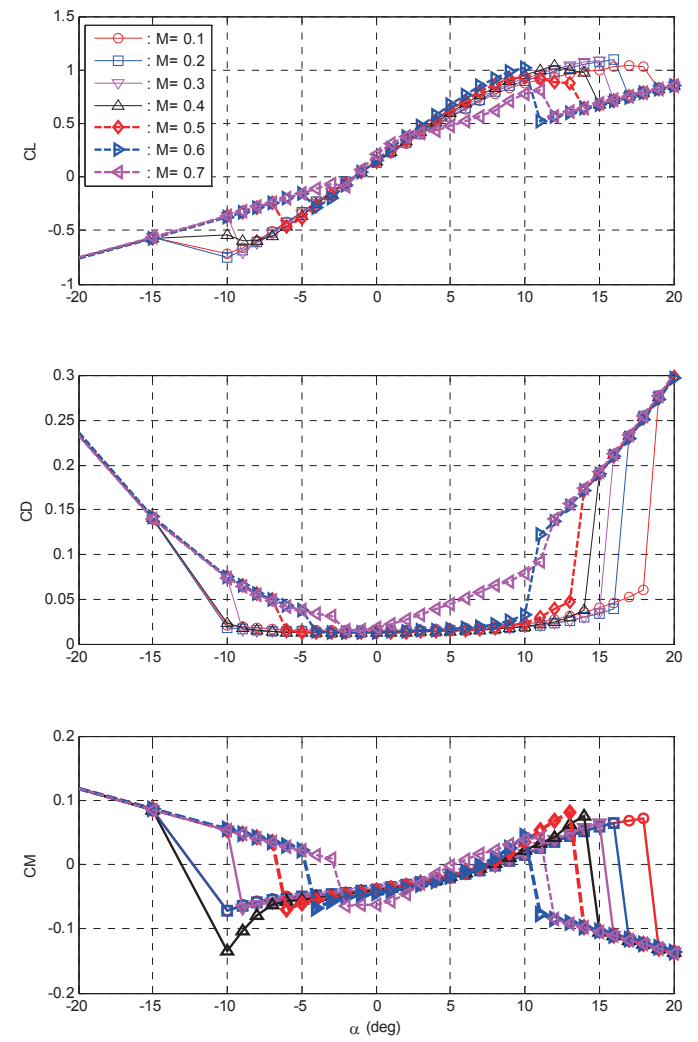

Fig. 3. Aerodynamic coefficients for the airfoil section used at the blade tip 


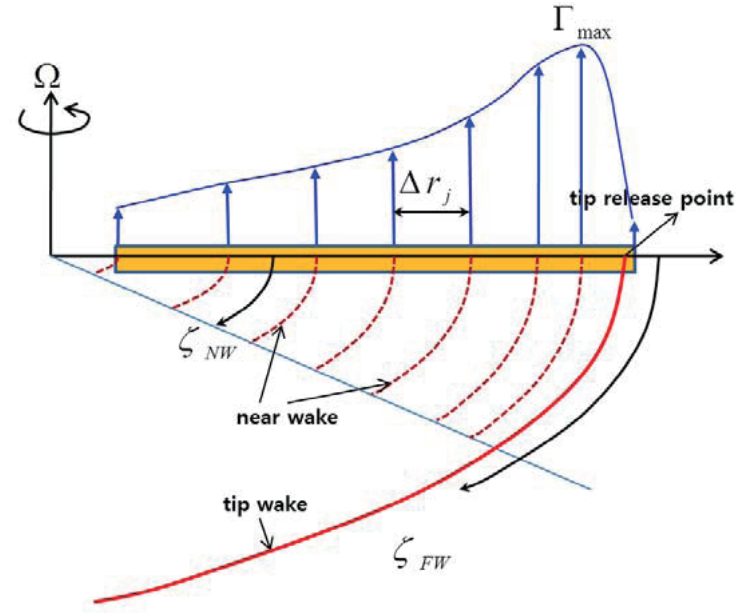

Fig. 4. Wake structure for the propeller aerodynamic model

ages $\left(\zeta_{F W} \leq 90_{0}\right)$ are used for the near wake filaments to save computing time. The strength of the tip vortex filament is defined using the single peak model [4] presented in Eq. (14) and the strengths of the near wake filaments can be determined by applying the conservation law of circulation as shown in Eq. (15) when $N_{\text {elem }}$ blade elements are used.

$$
\begin{gathered}
\Gamma_{\text {tip }}(\zeta)=\max \left(\Gamma_{b, j}\left(r_{b, j}, \psi-\zeta\right), j=1,2, \cdots\right) \\
\Gamma_{N W, j}= \begin{cases}\Gamma_{b, 1} & (j=1) \\
\Gamma_{b, j-1}-\Gamma_{b, j} & \left(j=2, \cdots, N_{\text {elem }}\right) \\
\Gamma_{b, N_{\text {elem }}} & \left(j=N_{\text {elem }}+1\right)\end{cases}
\end{gathered}
$$

The trajectories of the trailed wakes are determined using the prescribed wake model. By considering the flight speed, the wake geometry of each filament can be expressed as

$$
\mathbf{r}_{w}\left(\mathbf{r}_{b}, \psi, \zeta\right)=\mathbf{r}_{b}(\psi-\zeta)+\zeta \frac{\mathbf{V}_{F}}{\Omega}+\mathbf{D}\left(\mathbf{r}_{b}, \psi, \zeta\right)
$$

The first term $\mathbf{r}_{b}(\psi-\zeta)$ denotes the position vector where the vortex filament with the vortex age $\zeta$ originates from the blade with the azimuth angle $\psi$. The velocity $\mathbf{V}_{F}$ is the relative local air velocity vector with the angular motion of the airplane taken into account. Finally, the contraction effect of the wake can be modeled by the term $\mathbf{D}\left(\mathbf{r}_{b}, \psi, \zeta\right)$, which is generally modeled using the measured vortex geometries or using the empirical curve-fittings for analytical predictions. Two well-known empirical models exist: Landgrebe's and Kocurek and Tangler's prescribed wake models [11, 12]. These models incorporate both the radial contraction $D_{r}(r, \zeta)$ and the axial motion of the wake $D_{z}(r, \zeta)$, caused by the induced velocity. The resultant wake geometry can be represented using the empirical constants $k_{j}(j=1,2,3,4)$ as shown below

$$
\mathbf{D}\left(\mathbf{r}_{b}, \psi, \zeta\right)=D_{r}(r, \zeta) \cos (\psi-\zeta) \mathbf{i}+D_{r}(r, \zeta) \sin (\psi-\zeta) \mathbf{j}+D_{z}(r, \zeta) \mathbf{k}
$$

where $D_{r}(r, \zeta)$ and $D_{z}(r, \zeta)$ represent the shape functions for the wake geometry [4].

Landgrebe's model addresses both the tip vortex and the inboard vortex sheet, with the geometries represented using the thrust coefficient $C_{T}$ and the average twist angle $\theta_{r w}$ (in degrees). In addition, Kocurek and Tangler's model accounts for the blade number effect on the tip vortex geometry. The detailed model parameters can be found in Ref. [4] for both models. Once the strengths and geometries of all vortex filaments are determined, the induced velocities at any position can be computed using the Bio-Savart law. Vatistas' model [13] for the vortex core radius is adopted in the study to avoid the singular behavior of the induced velocity when the radial distance from a line vortex element approaches zero.

\section{Modeling the Propeller Effect on Airframe Aerodynamics}

The detailed aerodynamic models for the airframe can be found in Ref. [14]. The major approaches used to build the math model in this study will be summarized briefly for the sake of completeness. The aerodynamic forces and moments of the fuselage, main and tail wings are greatly affected by both the propeller's wake and the flow velocities induced by their lift forces. These effects can be modeled using the VLM [5] for flight dynamic analyses. The flow tangency conditions at the control points of the vortex lattices are typically imposed to compute the vortex strengths for all vortex filaments. By adding the effect of the airplane's angular motion when the flow tangency condition is imposed, maneuvering flights can be analyzed under the VLM framework. Although the VLM can accurately predict the lift and the pitching moment, even with a large angle of attack, it cannot account for viscous flow effects. To remedy this drawback, the VLM is used only to compute the induced velocity field and strip theory is applied to compute the aerodynamic forces and moments of the wing components. This approach might be effective for propeller-driven airplanes, in which wing components have a relatively high aspect ratio. Therefore, the viscous effect for the airfoil sections used in each wing can be easily considered with aerodynamic tables.

Figure 5 shows the striped wing elements for the main and tail wings. The local air velocity at the aerodynamic center of a wing element can be written as

$$
\mathbf{V}_{A C}=\mathbf{V}_{\infty}+\boldsymbol{\omega}_{C}^{C I} \times \mathbf{r}_{C}^{W C}+\mathbf{V}_{\text {Ind.prop }}+\mathbf{V}_{\text {Ind,airframe }}
$$




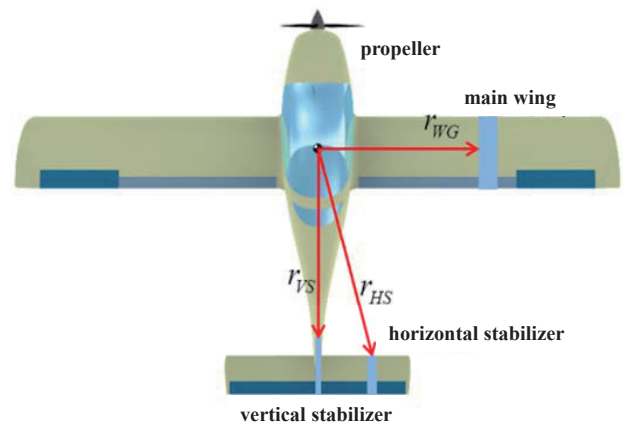

Fig. 5. Striped wing elements for the main and tail wings

where

$\mathbf{V}_{\infty}=u \mathbf{e}_{x}+v \mathbf{e}_{x}+w \mathbf{e}_{x}$

$\boldsymbol{\omega}_{C}^{C I}=p \mathbf{e}_{x}+q \mathbf{e}_{x}+r \mathbf{e}_{x}$

The vector $\mathbf{r}_{C}^{W C}$ represents the relative position $\left(\mathbf{r}_{W G}, \mathbf{r}_{H S}\right.$, $\mathbf{r}_{V S}$ ) of the striped wing element with respect to the airplane's CG. The velocity vectors, $\mathbf{V}_{\text {Ind.prop }}$ and $\mathbf{V}_{\text {Ind.airfame }}$ represent the induced velocities due to the propeller wake and the total downwash from other aerodynamic components, respectively. Fig. 6 shows the schematic diagram depicting the control point, the line elements of the bound vortex and the trailed wake. Fig. 7 shows the vortex lattices over the wings, fuselage, and control surfaces when VLM is applied to the light-sports airplane.

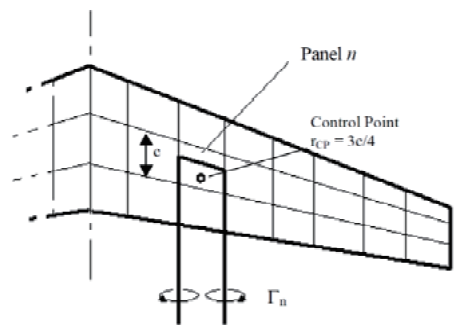

Fig. 6. Schematics for the VLM

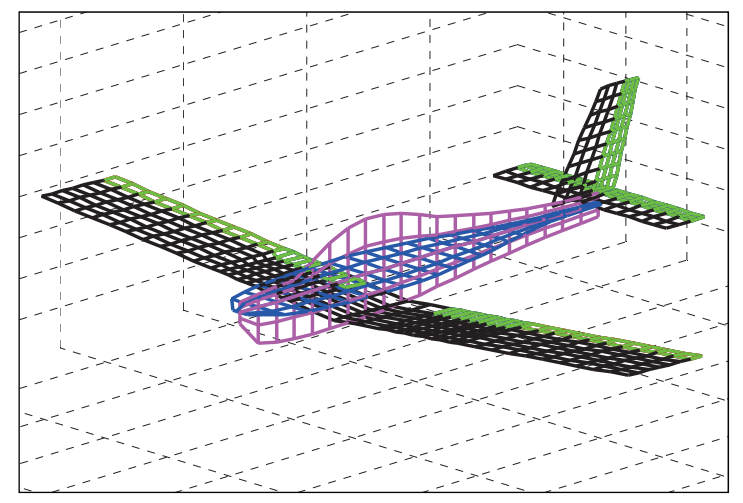

Fig. 7. Vortex lattice distributed over the aerodynamic surfaces for the VLM
The geometry of the trailing vortex filaments can be prescribed as in the propeller or traced using the free wake theory. Even though the geometry predicted using the free wake theory shows a complex structure after the rollup process as discussed in Ref. 4, its effect on the induced velocity fields over the wing components is nearly the same as when it is modeled with straight lines taking into account the flight velocity direction. Therefore, this paper uses the straight line vortex filament to reduce computational time. The air loads of each wing component are computed using 2-D aerodynamic tables as in the BEM. However, the aerodynamic forces and moments for the fuselage are modeled using the wind tunnel database rather than the values obtained from the table for the analysis with the VLM in order to include the viscous drag.

\section{Applications and Validation}

The propeller models proposed in this paper are applied to a light-sports-airplane, KLA-100, which is developed under the KAS-VLA certification requirements established by the Korean airworthiness certification authority. Table 1 shows the major performance and configuration data for the KLA-100 while Fig. 8 shows the distribution of the chord and the twist for the propeller. The installed 3-bladed propeller operates with a fixed pitch setting which can be adjusted on the ground. Using these data, the isolated propeller performances are first predicted with variations in the pitching settings and in the flight speed. Next, the proposed propeller models are implemented in the flight dynamic math model for the KLA-100, which is used to investigate the propeller effects on the air loads over major aerodynamic surfaces. Also, the effects of the gyroscopic moments generated by the propeller are investigated through trim analyses for the coordinated turn flights. Finally, maneuver simulations for the scaled KLA-100 model are performed with the control inputs used in flight tests to validate the proposed propeller models.

The propeller performance is predicted for the root pitch setting of 30 degrees at the sea-level ISA (International Standard Atmospheric) condition. The effects of flight speed and the propeller's RPM are shown in Fig. 9. The result shows that propeller efficiency is increased as RPM decreases, using the current setting of the root pitch angle over most of the speed range. The maximum thrust-to-weight ratio of over 0.33 can be obtained near the maximum RPM. In this condition, the negative rolling moment due to the propeller torque can be increased over $500 \mathrm{~N} \mathrm{~m}$.

The propeller wake effects on aerodynamic performance 


\begin{tabular}{l|l}
\hline \multicolumn{2}{c}{ Weight, Engine, and propeller } \\
\hline Max. take-off weight & $620 \mathrm{~kg}$ \\
Engine & $100 \mathrm{hp} \mathrm{Rotax}$ 912s2 \\
Propeller & 3 \\
- Number of blades & $1.726 \mathrm{~m}$ \\
- Diameter & 3000 \\
- Maximum RPM & \\
\hline
\end{tabular}

\section{Geometry of main wings(WG) and stabilizers}

\begin{tabular}{c|c|c|c}
\hline & WG & HS & VS \\
\hline Span (m) & 9.5 & 1.55 & 1.30 \\
\hline Chord (m) & 1.20 & 0.65 & 1.30 \\
\hline Twist angle (deg) & -3.0 & 0.0 & 0.0 \\
\hline Sweep angle (deg) & 0.0 & 0.0 & 10.0 \\
\hline Dihedral angle (deg) & 5.9 & 0.0 & 0.0 \\
\hline
\end{tabular}

\begin{tabular}{c|c|c|c|c}
\hline \multicolumn{6}{c}{ Geometry of control surface components } \\
\hline & flap & aileron & elevator & rudder \\
\hline Chord (\%) & 30 & 30 & 30 & 30 \\
\hline Spanwise & 0.72 & 3.39 & 0.0 & 0.0 \\
Location \\
$(\mathrm{m})$
\end{tabular}

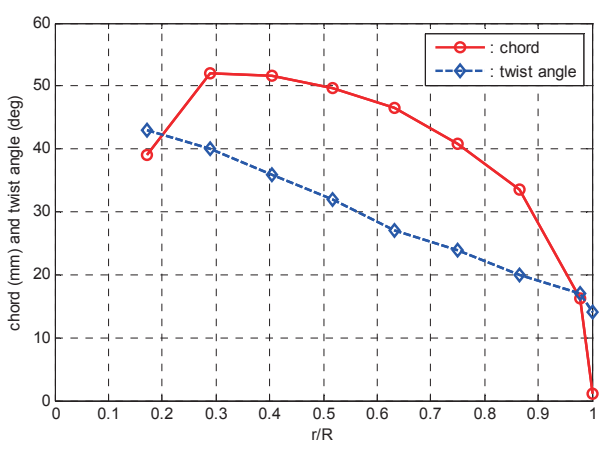

Fig. 8. Distribution of chord length and twist angle along the blade span
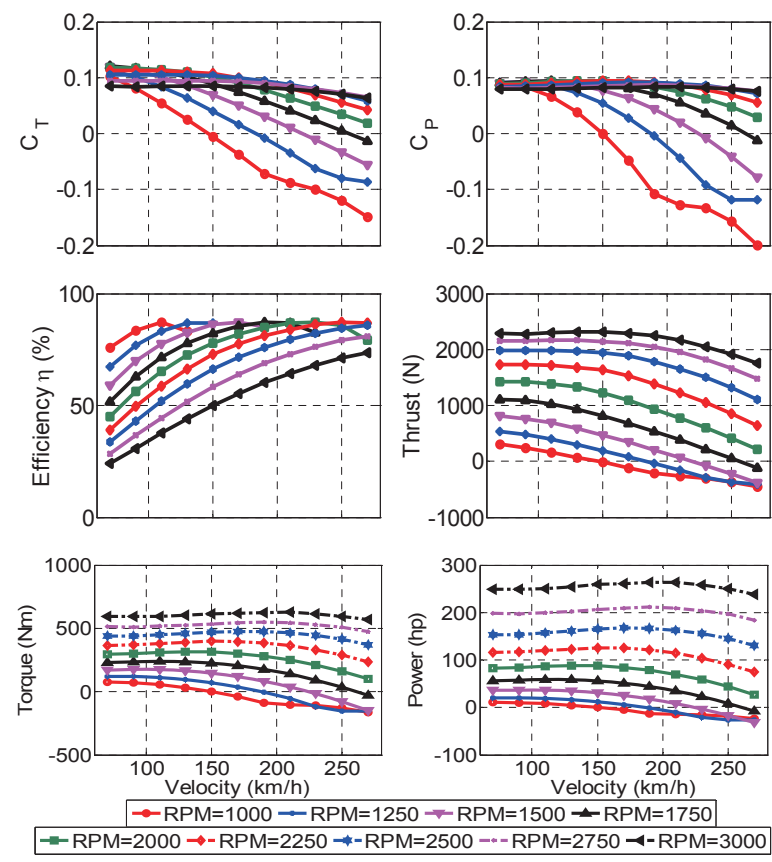

Fig. 9. Results of propeller performance prediction are studied though trim analyses for steady level flights with variations in flight velocity. For this purpose, the following four different cases were selected for comparative studies.

Case 1: using the coupled propeller and airframe aerodynamic model proposed in this study

Case 2: trim analyses without the propeller inflow effect on the airframe aerodynamics

Case 3: the same condition as Case 1 but the propeller torque effect is ignored

Case 4: the same condition as Case 2 but the propeller torque effect is ignored

Using the above combinations, the propeller torque effect can be identified from the results of analyzing Cases 1 and 3. Also, the propeller inflow effect can be deduced by comparing the results of Cases 1 and 2. Trim analyses were performed over the speed range of $100 \mathrm{~km} / \mathrm{hr}$ to $220 \mathrm{~km} / \mathrm{hr}$ at sea level standard ISA conditions with the maximum takeoff weight. Fig. 10 and 11 show the variations in trim attitude angles and the control inputs, respectively. The effect of the
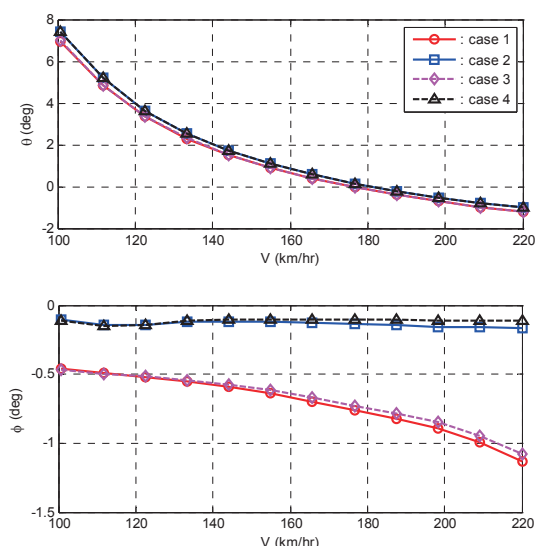

Fig. 10. Trim attitude angles (forward flight, sea level, standard ISA) 
propeller torque is relatively small compared to that of the propeller wake. The pitch attitude, the RPM, and the elevator deflection are negligibly affected by the propeller, unlike the roll angle and the lateral-directional controls which are considerably affected. The wake effects can be visualized by comparing the distributions of wing loadings as shown in Fig. 12. The root section of the main wing shows highly unsymmetrical distribution of the wing loadings which generates a large positive rolling moment. The tip region shows the effect of aileron deflection in compensating for the moment and maintaining steady level flight. In addition, the horizontal and vertical stabilizers have unsymmetrical wing loadings and the rudder deflection shows discernible differences in compensation for the yawing moment generated by the vertical stabilizer due to propeller induced velocity.

The effect of the gyroscopic moments resulting from the propeller rotation and the angular motions of the airplane are investigated through trim analyses of the coordinated turn flight, with variations in the normal load factor. Analyses are performed with a flight speed of $200 \mathrm{~km} / \mathrm{hr}$ and a normal load factor in the range of $n_{Z}=1.1 \sim 4.0$. The gyroscopic moments generated from the propeller are proportional to
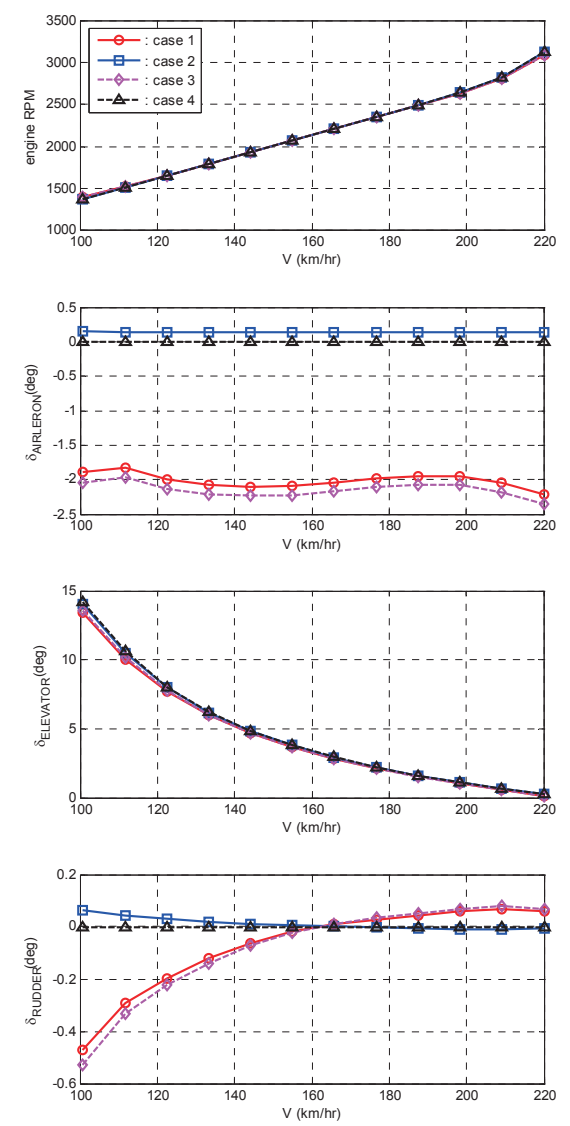

Fig. 11. Propeller RPM and controls for trimmed level flight the product of the body angular rate $\left(p_{S}, q_{S}\right)$ and $J_{b} \Omega$ as shown in Eq. (11). Their effects on general maneuvering flights can be deduced from the analyses by varying the propeller's polar moment of inertia. For this purpose, analyses are undertaken over the range of $J_{b}=0.0 \sim 6.0 \mathrm{~kg} \mathrm{~m}^{2}$, where the trim flight without the gyroscopic moment effect is defined by $J_{b}=0.0$. Fig. 13 represents the pitch, roll, and yaw rates during turn maneuvers and the pitch rate shows the largest variation. Therefore, the propeller gyroscopic moments mainly contribute to the pitching moment around the airplane's CG for the level turn flight. Figs. 14 and 15 present the body attitudes and controls. The rudder deflection $\delta_{\text {rudder }}$ with $J_{b}=6.0$ $\mathrm{kg}-\mathrm{m}^{2}$ is around 7 degrees less than that required with $J_{b}=0.0$ for the turning flight, with $n_{Z}=4.0$. Therefore, the gyroscopic moment can significantly affect the control margin. It can also degrade maneuverability by decreasing the propeller efficiency as shown in Fig. 16. The polar moment of inertia $J_{b}$ for the VLA-100 is estimated to be around $0.75 \mathrm{~kg}-\mathrm{m}^{2}$. Therefore, $J_{b}=6.0$ seems to be unrealistically high. The VLA100 must be maneuverable at over 180 degrees/sec pitch rate during aggressive maneuvers. Therefore, the present analyses with a large $J_{b}$ can be used to estimate the effect of
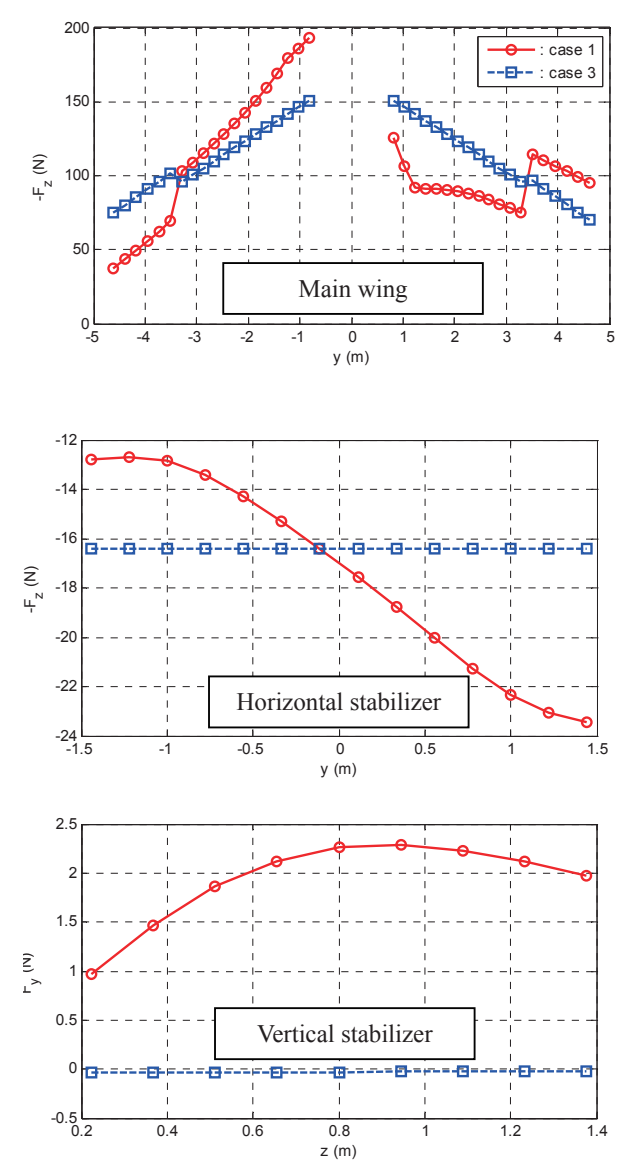

Fig. 12. Propeller effects on the distribution of wing loading 

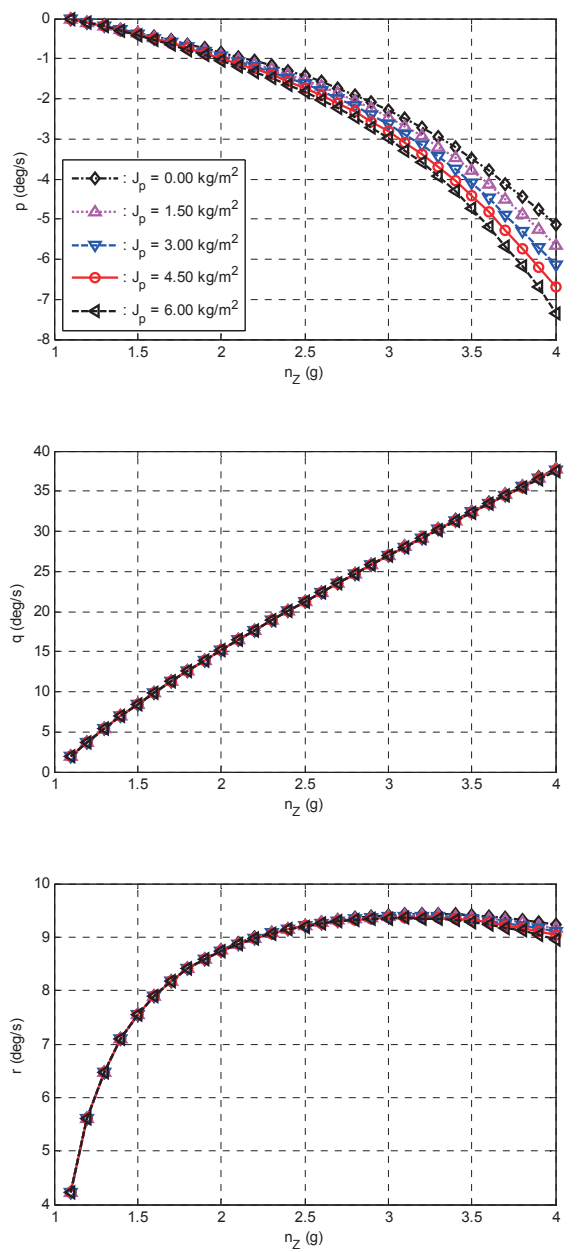

Fig. 13. Angular rates for trimmed turn flights
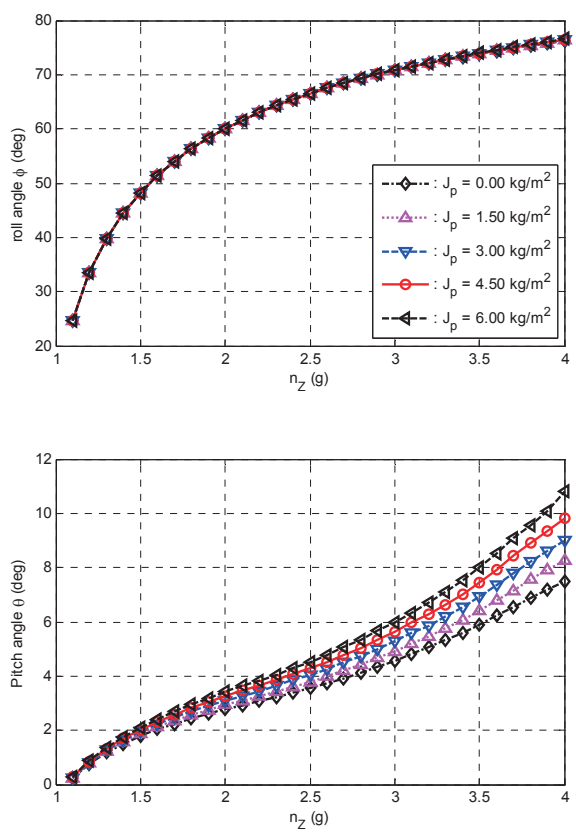

Fig. 14. Attitude angles for trimmed turn flights the propeller's gyroscopic moments in real flights.

The proposed propeller models were applied to the simulation studies and the results were compared with the flight test data collected from the 1/5-scale RC model of the KLA-100. Fig. 17 shows the RC model with the two-bladed propeller on the runway ready for flight tests. A series of flight tests were carried out with varying propeller thrust conditions. The results of the control response tests at $80 \%$ of
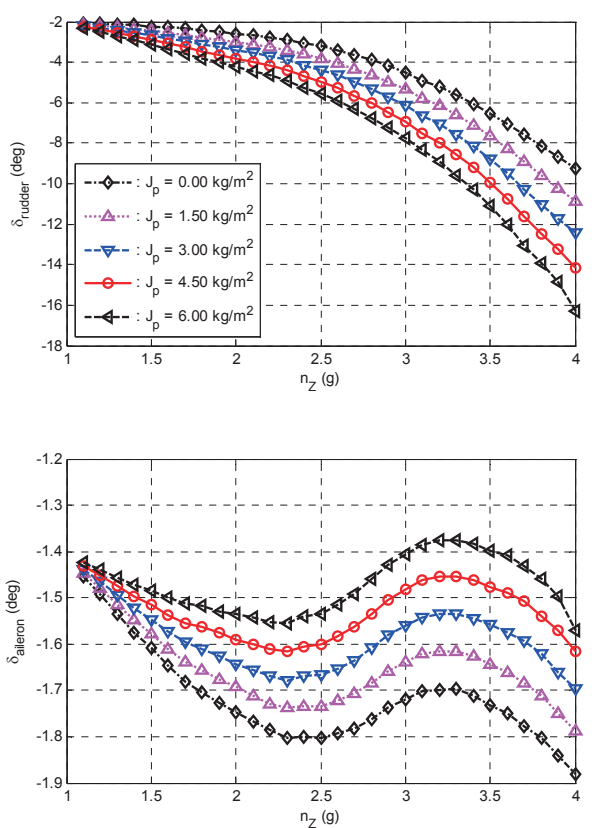

Fig. 15. Rudder and aileron deflections for trimmed turn flights
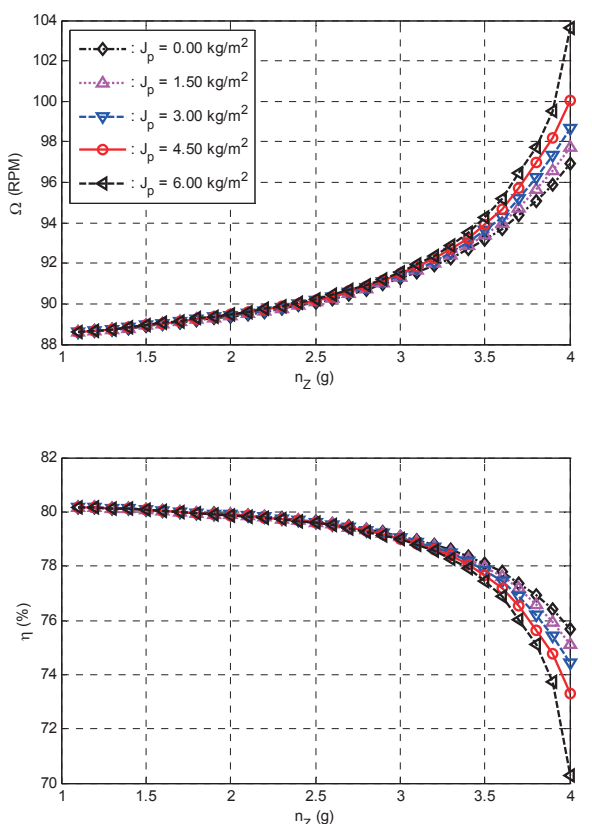

Fig. 16. RPM and propeller efficiency during trimmed turn flights 


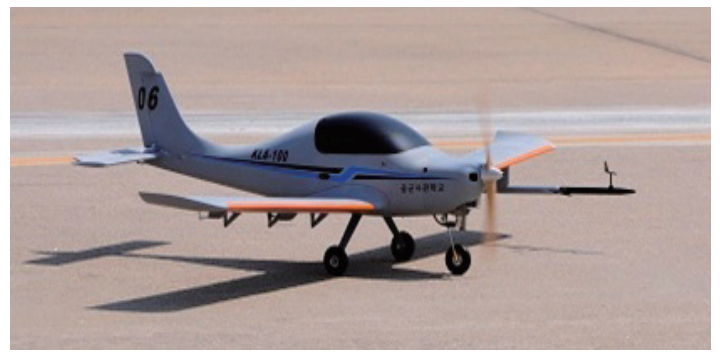

Fig. 17. Scaled RC-model of KLA-100 for flight tests

the maximum propeller thrust are compared to validate the proposed propeller model. Figs. 18-20 compare the results of the flight simulations with those of the corresponding flight tests. The doublet-type input was imposed for each of the control channels. The relevant responses are represented in the attitude angle and the angular rate. The RC model is equipped with only the attitude and heading reference system (AHRS), containing a coarse alignment of its axes with the reference frame of the airplane due to space limitations. Therefore, the effects of the relative wind velocity and the misalignment in the AHRS reference frame
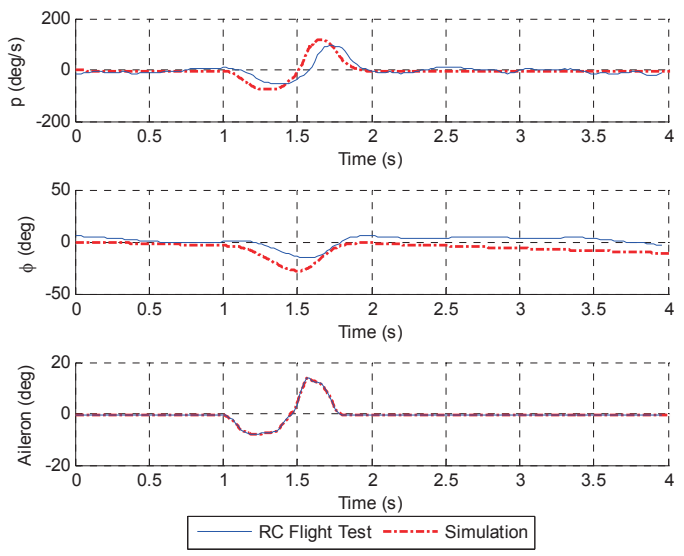

Fig. 18. Response comparison for aileron doublet input at $0.8 T_{\max }$
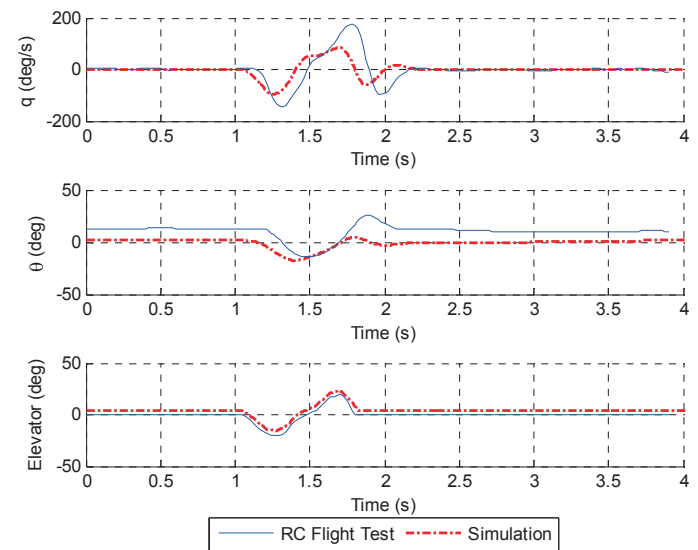

Fig. 19. Response comparison for elevator doublet input at $0.8 T_{\max }$
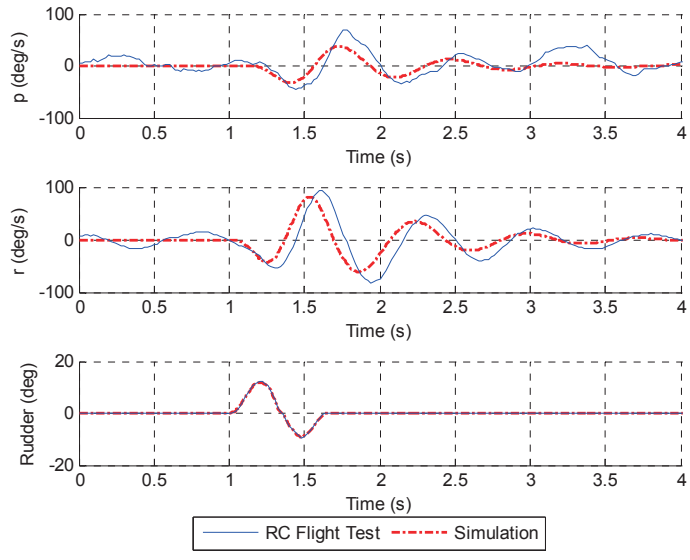

Fig. 20. Response comparison for rudder doublet input at $0.8 T_{\max }$

cannot be duly accounted for in the measurements of the trim attitude angles. Also, the configuration of the RC model may differ from the actual design, especially with respect to the propeller and control surface geometries. Therefore, the measured trim attitudes, which are shown at the initial time for each figure, are discernibly different from the computed ones. However, the overall dynamic responses to the doublet inputs for each channel show a good correlation between the two datasets, with a small phase shift due to the delay in the communication link.

\section{Conclusions}

This paper focuses on the aerodynamic and inertial modeling of the propeller in order to build a high-fidelity math model for propeller-driven airplanes. The effects of the trailing wake on other aerodynamic surfaces were successfully modeled using the blade-element-method combined with the vortical wake theory. The gyroscopic moments of the propeller were formulated in an exact manner from the kinematics, with all body states taken into account. In addition, the vortex-lattice method was adopted to consider the velocity induced by the propeller while strip theory was utilized to compute the forces and moments generated by the wing components. Therefore, the resultant aerodynamic models can accurately address the compressibility effect over the propeller blade and viscous effects on air loads for all aerodynamic surfaces. These models were integrated into the flight dynamic analysis program for a light-sports-airplane KLA-100 and various applications were tested to validate the usefulness of the proposed approaches.

The roll attitude and lateral-directional control are significantly affected by the propeller wake for the trimmed 
level forward flight, whereas the propeller torques have relatively small influences on the trim states and controls. The effect of the gyroscopic moments generated by the propeller was investigated through a series of trim analyses for the coordinated turn flight, with variations in the polar moment of the propeller. The large pitch rates required for turn flights with a high normal load factor mainly generates the gyroscopic moment around the airplane's yaw axis, which causes large rudder deflections. Flight simulations were undertaken for the scaled KLA-100 model and the results were compared with the corresponding flight test data. The airplane responses to doublet inputs for the aileron, elevator, and rudder deflections using the methods presented are well correlated with the flight test results. The applications show that a high-fidelity math model for a propeller-driven airplane can be built using the proposed aerodynamic and inertial models for the propeller.

\section{Acknowledgments}

This research was supported by a grant (1615001723) from the Light Aircraft Development Program funded by the Ministry of Land, Infrastructure and Transport affairs of the Korean government.

\section{References}

[1] TaeSan Park, Chang-Joo Kim, Sanh Hoon Shin, and Sunguk Hur, "Analysis Techniques of Flight Performance and Stability Analysis for Light Sports Airplane," The Korean Society for Aeronautical and Space Science, Spring conference, April, 2012.

[2] Gareth D. and Padfield, Helicopter Flight Dynamics, Blackwell Science, 2007.

[3] Yun, Y.-H., Kim, C.-J., Shin, K.-C., Yang, C.-D. and Cho, I.-J., "Building the Flight Dynamic Analysis Program,
HETLAS, for the Development of Helicopter FBW System," $1^{\text {st }}$ Asian Australian Rotorcraft Forum and Exhibition 2012, Bexco, Busan, Korea, February 12-15, 2012.

[4] J. Gordan Leishman, Principles of Helicopter Aerodynamics, Cambridge University Press, 2006.

[5] John D. Anderson, Jr., Fundamentals of Aerodynamics, McGraw Hill, 2011.

[6] Chang-Joo Kim, "Implicit Formulation of Rotor Aeromechanic Equations for Helicopter Flight Simulation," Journal of KSASS, Vol. 33, No. 3, April 2002, pp. 8-16.

[7] Chang-Joo Kim, "Helicopter Trim Analysis by Using Level 2 Mathematical Model for Rotor," The Korean Society for Aeronautical and Space Science, Autum conference, December 2001.

[8] Park, S.H. and Kwon, J.H., "Implementation of Turbulence Models in an Implicit Multigrid Method," AIAA Journal, Vol.42, No.7, 2004, pp.1348-1357.

[9] Lee, S.M., Sa, J.H., Jeon, S.E., Kim, C.J., Park, S.H. and Chung, K.H., "A Response-Surface Methodology for Rotor Airfoil Design with Multiple Design Constraints," 36th European Rotorcraft Forum, Paris, France, 2010.

[10] Bonilla, C., Gompertz, K., Thake, M., Bons, J.P., Park, S.H. and Kim, C.J., "Effect of Thickness Distribution on Airfoil Performance Near Critical Mach Number," AIAA Aerospace Sciences Meeting, Nashville, Tennesse,2012.

[11] Landgrebe A. J., "An Analytical Method for Predicting Rotor Wake Geometry," Journal of the American Helicopter Society, Vol. 14, No. 4, 1969, pp.20-32.

[12] Kocurek J. D. and Tangler J. L., "A Prescribed Wake Lifting Surface Hover Performance analysis," Journal of the American Helicopter Society, Vol. 21, No. 1, 1976, pp.24-35.

[13] Vatistas G. H. , Kozel V. and Mih W.C., "A Simpler Model for Concentrated Vortices," Experiments in Fluids, Vol. 11, 1991, pp. 73-76.

[14] Chang-Joo Kim, et. al., "Flight Dynamic Analyses of Propeller-Driven Airplane (II): Building a High-Fidelity Math Model and Applications," International Journal of Aeronautical and Space Sciences, Vol. 15, No. 4, 2014, pp. 356-365. 